\title{
Editorial
}

\section{Inter- and Intra-examiner Variability of a Measure and Its Clinical Importance}

\author{
Gary Jacobson, ${ }^{1}$ Editor-in-Chief, Journal of the American Academy of Audiology \\ ${ }^{1}$ Department of Hearing and Speech Sciences, Vanderbilt \\ University Medical Center, Nashville, Tennessee
}

J Am Acad Audiol 2020;31:551-552.

The contemporary vestibular test battery consists of either monothermal or bithermal caloric testing, computerized sinusoidal harmonic acceleration (SHA) (i.e., the rotary chair test), and video head impulse test (i.e., the vHIT test). The eras when these assessments were developed for clinical purposes were (arguably) the 1940s, the 1970s, and the beginning of the current century.

Each test evaluates the performance of the vestibular system over a different portion of the frequency spectrum of the vestibular system, with the caloric test being most useful in the assessment of function at approximately $0.003 \mathrm{~Hz}$. The SHA test assesses the performance characteristics of the vestibular system over the frequency range 0.01 to $0.32 \mathrm{~Hz}$ and the vHIT test assesses function over a frequency range that is encountered in everyday life, from approximately $1 \mathrm{~Hz}$ to approximately $6 \mathrm{~Hz}$. Since the first stage of central compensation following unilateral impairment is sacrificing velocity storage (a low-frequency event), it is common to identify the presence of unilateral impairment by using caloric testing.

One of the steps in the development of a new measure is the assessment of its intra-examiner variability. That is, how variable are the results of a measure for a single individual over multiple administrations. Another approach would be to assess the inter-examiner variability of a measure. In this case, we measure the variability of a measure for multiple individuals and for a single administration.

It is also important to assess the test/retest reliability of a new measure. The information gleaned from those studies tell investigators, among other things, how large of a net change in score from test to test is necessary for the change to be clinically significant.

I am focusing this editorial on a paper titled "Evaluation of Inter- (vs. Intra-) Examiner Variability in Video Head Impulse Test (vHIT) Results" by Mutlu and colleagues. Now, there are many sources of variability in the vHIT. For example, the technique for holding the head is a source of variability, as is the position of the examiner's hands on the strap and the potential for movement. Other sources of variability include the effect of direct contact between the goggle and the examiner's hand, head bounce, and even inadequate pupil-targeting may contribute to variability in the test results.

For this study, the investigators conducted their measurements on 21 "healthy participants." The vHITs were conducted by four clinicians. The dependent variables were VOR gain values. I am not going to provide a "spoiler" here, except to say that there were significant inter-examiner differences. Please enjoy this issue of JAAA.
Copyright $\odot 2020$ by the American Academy of Audiology. All rights reserved. Thieme Medical Publishers, Inc., 333 Seventh Avenue, New York, NY 10001 , USA.

Tel: +1(212) 760-0888.
DOI https://doi.org/ 10.1055/s-0040-1721803. ISSN 1050-0545. 
